\title{
Phase behavior of hard spherical caps
}

\author{
Giorgio Cinacchia) \\ Departamento de Física Teórica de la Materia Condensada and Instituto de Física de la Materia Condensada, \\ Universidad Autónoma de Madrid, Campus de Cantoblanco, E-28049 Madrid, Spain
}

(Received 30 June 2013; accepted 26 August 2013; published online 30 September 2013)

\begin{abstract}
This work reports on the phase behavior of hard spherical caps in the interval of particle shapes delimited by the hard platelet and hemispherical cap models. These very simple model colloidal particles display a remarkably complex phase behavior featuring a competition between isotropicnematic phase separation and clustering as well as a sequence of structures, from roundish to lacy aggregates to no ordinary hexagonal columnar mesophases, all characterized by groups of particles tending to arrange on the same spherical surface. This behavior parallels that one of many molecular systems forming micelles but here it is purely entropy-driven. (C) 2013 AIP Publishing LLC. [http://dx.doi.org/10.1063/1.4822038]
\end{abstract}

The self-assembly of molecular and colloidal systems has always, and nowadays especially, attracted much attention. ${ }^{1}$ Recently, in the case of colloids, there has been significant progress in the synthesis of particles of different shape and size as well as in the techniques to visualize the structures they form. ${ }^{2}$ These experimental advances offer concrete chances to observe those phase behaviors predicted by theory and numerical simulation; new results from these can in turn stimulate further experimental research. ${ }^{3}$ One current example is provided by hard polyhedral particles, to which much numerical and experimental effort is being dedicated. ${ }^{4-6}$

In theory and simulation, colloidal particles are indeed often assumed to interact through hard-body interactions as these are predominant in directing their packing and shown over the years to be sufficient for the stabilization of a variety of entropy-driven complex fluid phases: nematic $(\mathrm{N}){ }^{7}$ smectic, ${ }^{8}$ columnar (C), ${ }^{9}$ and even cubic gyroid ${ }^{10}$ phases can all be obtained in systems of hard-body particles of suitable shape and size.

This work considers a special class of hard-body model colloidal particles and examines by numerical simulation their phase behavior featuring both phase separation and aggregation phenomena.

The particles are hard spherical caps (HSCs); a preliminary account of their intriguing phase behavior has been given in Ref. 11. Each of these particles is the portion of the surface of a sphere of radius $R$ subtended by an angle $\theta$. The area of this portion is set equal to $\sigma^{2}$, with $\sigma$ the unit of length; any particle of this type is thus identified by $R^{*}=R / \sigma$ (or $\theta$ ). By varying $R^{*}$, hard, generally concave and infinitely thin, particles can be obtained going from the hard platelet, $\left(R^{*}\right.$ $\rightarrow \infty)$, through the hard hemispherical cap $\left(R^{*}=1 / \sqrt{2 \pi}\right)$, to the hard sphere $\left(R^{*}=1 / 2 \sqrt{\pi}\right)$ models. It is actually those lens-, bowl-, and vase-like particles that lay in between these limits that are most interesting.

HSCs fit current interest for a variety of reasons. These are primarily fundamental but also related to the issues of

$\overline{{ }^{a)} \text { Electronic mail: giorgio.cinacchi@ }}$ uam.es practical relevance, given, e.g., the wide range of possibilities that nano-sized hollowed particles may offer in catalysis and drug delivery. ${ }^{12}$ Several are the viewpoints HSCs can be regarded from.

(i) They are a generalization of the hard infinitely thin platelet model, ${ }^{13}$ the basic model to study discotic liquid crystals. This model is the counterpart of the hard long and thin rod model. While the latter is exactly described by the Onsager second-virial density functional theory, ${ }^{7}$ higher order virial terms are, however, not negligible for hard platelets ${ }^{7,13}$ and one has to attempt other theoretical approaches (e.g., Refs. 14 and 15).

(ii) They are concave, either lens- or bowl- or vase-like, particles. Theoretical and simulational studies on the phase behavior and properties of systems of convex anisotropic particles, hard and soft, are many. ${ }^{16,17}$ One study, very recent and quite related to the present work, investigates the phase behavior under confinement of hard particles consisting of a sphere cut off by a plane at a certain height. ${ }^{18}$ Hard or soft concave particles have been far less studied. To date, the most studied concave particles have an arched shape. The interest in them, stirred by the discovery of particular ferroelectric phases that molecules with a bent core give rise to, ${ }^{19}$ has been more recently reinvigorated by claims that certain of these molecules have a biaxial nematic phase, ${ }^{20}$ though the effects observed look more likely due to the presence of cybotactic clusters. ${ }^{21}$ Very fewer studies have been performed on systems of concave particles having a lensor bowl-like shape. Beside Ref. 11, two more simulation studies ${ }^{22,23}$ have been published recently. Both of these studies consider bowl-like particles and focus on the columnar phases that they can form. HSCs provide a simpler model for molecular ${ }^{24,25}$ and colloidal ${ }^{26}$ bowlic liquid crystals as well as being a first, minimal representation of metallic (half-)shells ${ }^{27}$ and of the very interesting buckybowlic molecules, concave fragments of fullerene, whose curvature and rigidity may be chemically controlled. ${ }^{28}$ 
(iii) They form an entropy-driven cluster phase, the colloidal analogue of the micellar phase in surfactant solutions, and may constitute a very simple model for the study of arrested states of matter. The possibility to regulate the shape of a HSC particle from plate- to lens- and bowllike, together with its zero volume are at the heart of the phase behavior reported in Ref. 11. In that Letter, it was shown that HSCs with such a curvature that resemble contact lenses $\left(R^{*}=1\right)$ do not form, on increasing density, a $\mathrm{N}$ phase, as flatter HSCs $\left(R^{*} \geq 2\right)$ do. Rather, for contact lens-like particles, the ordinary isotropic phase spontaneously evolves towards a phase organization with a locally favored structure ${ }^{29}$ characterized by lens-like particles tending to arrange on the same spherical surface, that is the centers of their parent spheres tend to aggregate. The fact that a cluster phase is observed in a pure system of hard particles is remarkable as in this case this sort of phase, usually the result of competing attractive and repulsive interactions ${ }^{30-34}$ or made possible by letting spherically symmetric particles overlap, ${ }^{35}$ is instead stabilized by the sole entropy. What is occurring in contact lens-like particle systems is a kind of geometric frustration; mechanisms of this type have long been thought at the origin of the arrested states of matter. ${ }^{36}$

With these premises, it would be of interest to sketch the whole phase diagram of HSCs as a function of $R^{*}$. To achieve this, one can start from previous results on lens-like particles ${ }^{11}$ and then interpret the formation of the worm-like structures reported in the high-density region of the fluid phase of hard hemispherical caps, which subsequently coexisted with an ordinary columnar phase, ${ }^{23(b)}$ as an echo of the aggregation behavior previously observed for lens-like particles. One can thus hypothesize that the aggregates formed by HSCs of sufficiently small curvature will form, on further compression, filamentous structures and then, from these, columnar phases whose basic unit is, however, not a single particle, as in the ordinary cases, included the hard hemispherical cap model, but a group of particles tending to arrange on the same spherical surface. This behavior may be thought of as paralleling that one of many molecular micellizing systems where the surfactants first form globular aggregates, the micelles, and then, on increasing their concentration, organize further into rod-like micelles and eventually columnar phases. ${ }^{37,38}$ The object of this work is to provide an evidence for this scenario.

The technique used is isobaric-isothermal Monte Carlo computer simulation, MC-NPT, ${ }^{39,40}$ with $\mathrm{N}$ the number of particles, $P$ the pressure, and $T$ the temperature. The basic element of these calculations was the overlap criterion between two HSCs: the one employed is consistent with that clearly described in Ref. 41 and able to recover the overlap criteria for hard platelets and hard spheres in their respective limits. Usually, cuboidal periodic boundary conditions were used but, on occasion, truncated-octahedral and variable-shape triclinical computational boxes were also implemented. Every simulation run was organized in cycles, each of them on average consisting of: $2 \mathrm{~N}$ attempts to translate or rotate a randomly selected particle; one attempt to invert the orientation of a randomly selected particle while keeping the position of its pole; and one attempt to vary the volume of the computational box by varying one, randomly selected, edge. The sequence of the attempted moves was random to preserve the microscopic detailed balance condition. Maximal displacement, angle of rotation and box edge variation were monitored to check that their respective acceptance ratios were around $20 \%-30 \%{ }^{42}$ and then accordingly adjusted, if needed, once a run had completed. Two (pseudo)random number generators were used: either ran $2,{ }^{43}$ mostly employed in those simulation runs with a relatively small $(\leq 1000) \mathrm{N}$, or mt $19937,{ }^{44}$ otherwise, finding the desired independence of the results on the pseudo-random number generator used.

HSCs belonging to the interval $R^{*} \in[1 / \sqrt{2 \pi} ; \infty)$ were considered. Their equation of state was calculated and the phases formed identified mostly by direct visualization using the program QMGA. ${ }^{45}$ Full quantitative analysis of their features along the lines presented in the previous work ${ }^{11}$ is deferred to a subsequent paper. Specifically, the values of $R^{*}$ investigated were: $1 / \sqrt{2 \pi}, 0.45,0.55,0.7,1,1.5,1.6,2,5$, 10,100 . The samples considered had a number of particles ranging from $O\left(10^{2}\right)$ to $O\left(10^{4}\right)$. Every state point investigated was identified by a fixed value of pressure measured in reduced units: $P^{*}=P \sigma^{3} /\left(k_{B} T\right), k_{B}$ being the Boltzmann constant. The configurations used to initiate an equilibration run can be grouped in two broad categories: (i) either a low density simple cubic lattice configuration, that rapidly melted, or a configuration generated during a run performed at a nearby state point or a configuration obtained by replicating twice in each direction a configuration obtained at the end of a prior run conducted with a smaller size sample; (ii) a hexagonal columnar configuration, either ordinary, in which particles are stacked on top of one another in a column and the columns are arranged in a hexagonal lattice or, as shown in Fig. 1, a configuration formed by hexagonally arranged columns whose basic units are however flocks of particles tending to share the same spherical surface. Typical runs were of the order of $O\left(10^{6}\right)$ up to runs of $O\left(10^{8}\right) \mathrm{MC}$ cycles. The density, $\rho$, was measured in reduced units: $\rho^{*}=\rho \sigma^{3}$. The nematic order parameter, $\left\langle P_{2}\right\rangle$, was also calculated and this was done in the usual way. ${ }^{46}$

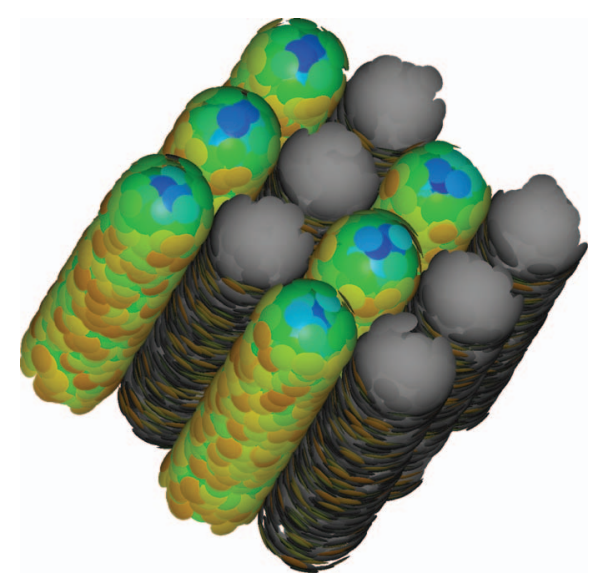

FIG. 1. Example of a hexagonal cluster columnar phase. 

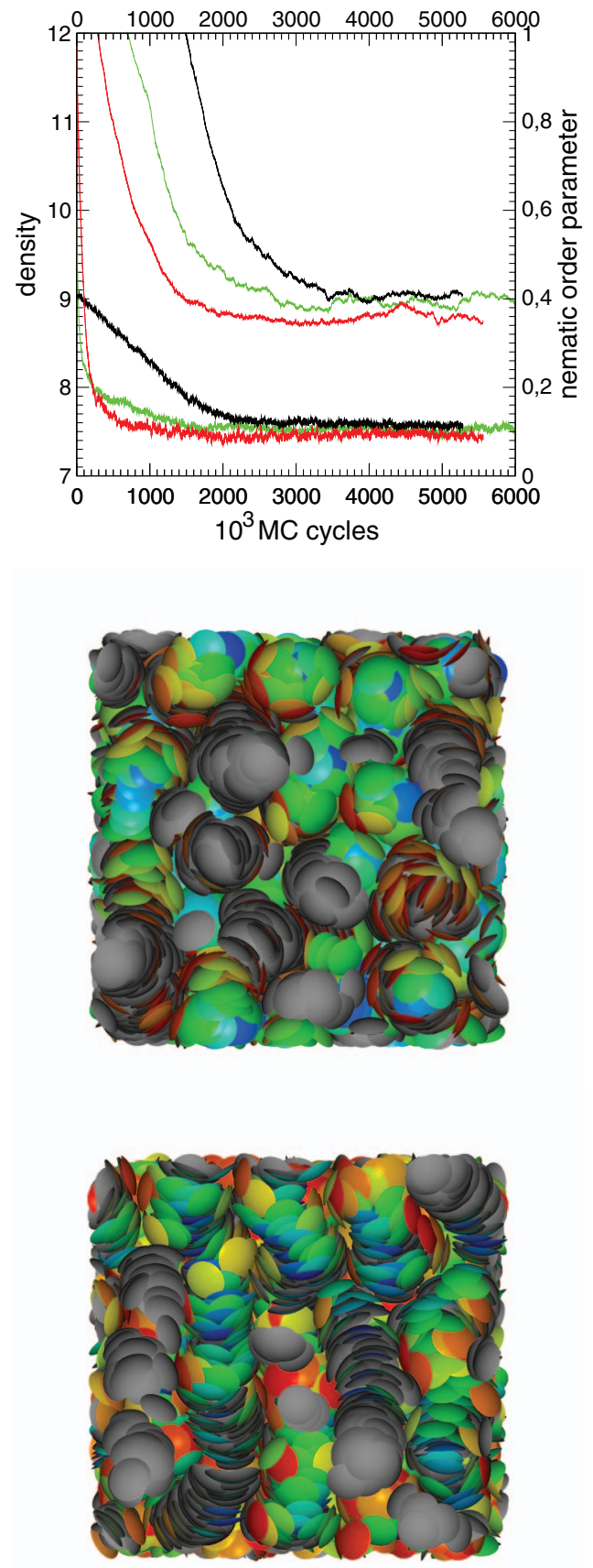

FIG. 2. (Upper panel) The evolution of a sample of 8000 particles with $R^{*}=1$ starting from different configurations: hexagonal single-particle columnar configuration with all particles pointing to the same direction (green or light gray curves); hexagonal single-particle columnar configuration with columns pointing up and down (red or dark gray curves); hexagonal cluster columnar configuration (black curves). Density refers to the left ordinate axis and its evolution corresponds to the top curves; nematic order parameter refers to the right ordinate axis and its evolution corresponds to the bottom curves. (Central and lower panels) Images of the system that started from the hexagonal single-particle columnar configuration with columns pointing up and down taken after $4.5 \times 10^{6}$ of MC cycles.

One element in support of the above-mentioned hypothesis that aggregates of HSCs will further organize, upon densification, into filaments and columns, is the spontaneous evolution, at $P^{*}=30$, of ordinary hexagonal single-particle columnar configurations, having either all columns pointing to the same or to opposite directions, of 8000 particles with $R^{*}=1$ towards a cluster phase. Figure 2 shows the evolution of $\rho^{*}$ and $\left\langle P_{2}\right\rangle$ along with snapshots ${ }^{47}$ obtained after several millions of MC cycles. This figure also shows the evolution of these properties starting from a moderate density hexagonal cluster columnar $\left(\mathrm{CC}_{h}\right)$ configuration like the one in Fig. 1. Irrespective of the starting configuration, equilibrium values of $\rho^{*}$ and $\left\langle P_{2}\right\rangle$ are consistent. From the two images it is apparent how particles clump; remnants of a columnar structure are retained though columns are irregular, rather tortuous and broken at certain points. One may look at this phase as a defected cluster columnar phase and imagine that a less defected phase of this type can form at higher pressure. With this piece of evidence, other values of particle curvature $R^{*}$ were investigated, starting simulation runs with configurations belonging to the above-mentioned two categories. Figure 3 shows the equations of state (EoS) obtained.

For the case with $R^{*}=2$ in Fig. 3(a) three branches can be distinguished: the low-density isotropic (I) branch, separated from the $\mathrm{N}$ branch by a weak first-order phase transition, and a higher density $\mathrm{CC}_{h}$ branch. The $\mathrm{N}$ phase is stable in the interval $P^{*} \in[14-19]$; its branch can be continued to higher pressure values though the corresponding equilibrium density value becomes progressively smaller than the one reached by the $\mathrm{CC}_{\mathrm{h}}$ phase. These two phases should be separated by a first-order phase transition. By starting a run from a well equilibrated $\mathrm{N}$ configuration at high density, there was no indication of a direct formation of a $C_{h}$ phase. Systems with 2000 particles prepared in a $\mathrm{CC}_{\mathrm{h}}$ configuration with $\rho^{*}=5$ rapidly melted to a $\mathrm{N}$ phase at $P^{*}=19$ but evolved towards a denser $\mathrm{CC}_{\mathrm{h}}$ phase at $P^{*}=22$ and larger values of pressure. It was necessary to start with a $\mathrm{CC}_{\mathrm{h}}$ configuration with a sufficiently high density, $\rho^{*}=5$, in order to see the system compress while keeping a $\mathrm{CC}_{\mathrm{h}}$ structure. Otherwise, the system evolved towards a $\mathrm{N}$ phase even at high pressure. On one occasion, however, starting a run at $P^{*}=30$ from a $\mathrm{CC}_{\mathrm{h}}$ configuration of 6000 particles with $\rho^{*}=4.6$, it was observed that the system initially acquired a $\mathrm{N}$ structure, with a density $\rho^{*} \simeq 6$ and instantaneous $\left\langle P_{2}\right\rangle \simeq 0.7$, but then spontaneously evolved to a complex bunchy structure in which particles aggregate and seemingly form tortuous thick filaments, and having $\rho^{*} \simeq 9.3$ and $\left\langle P_{2}\right\rangle \sim 0.06$, although being then unable to fully develop in to a $\mathrm{CC}_{\mathrm{h}}$ phase (Figs. 4(a) and 4(b)). Though small, there is a difference between the density reached by this irregular bunchy structure and the value reached by starting a run from a pre-built $\mathrm{CC}_{\mathrm{h}}$ configuration, with the former being $\sim 7 \%$ larger. This difference could be due to the different system size but a simulation run with $\mathrm{N}=12000$ particles that started from a $\mathrm{CC}_{\mathrm{h}}$ configuration also ended up to a density of 8.65 , consistent with that of the system with $N=2000$ and thus smaller than that reached by the irregular bunchy structure of Figs. 4(a) and 4(b). Similar results are obtained at smaller pressure, $P^{*}=21$ and 25 , and for systems with $\mathrm{N}$ $=4096$, for which the same type of bunchy structures is observed having essentially the same density of the $C_{h}$ phase, with an overall very small nematic order parameter. This is comprehensible as the $\mathrm{CC}_{\mathrm{h}}$ phase and the complex bunchy structures have both the same local structure and the latter dictates the EoS in a hard-body particle system. It cannot be 

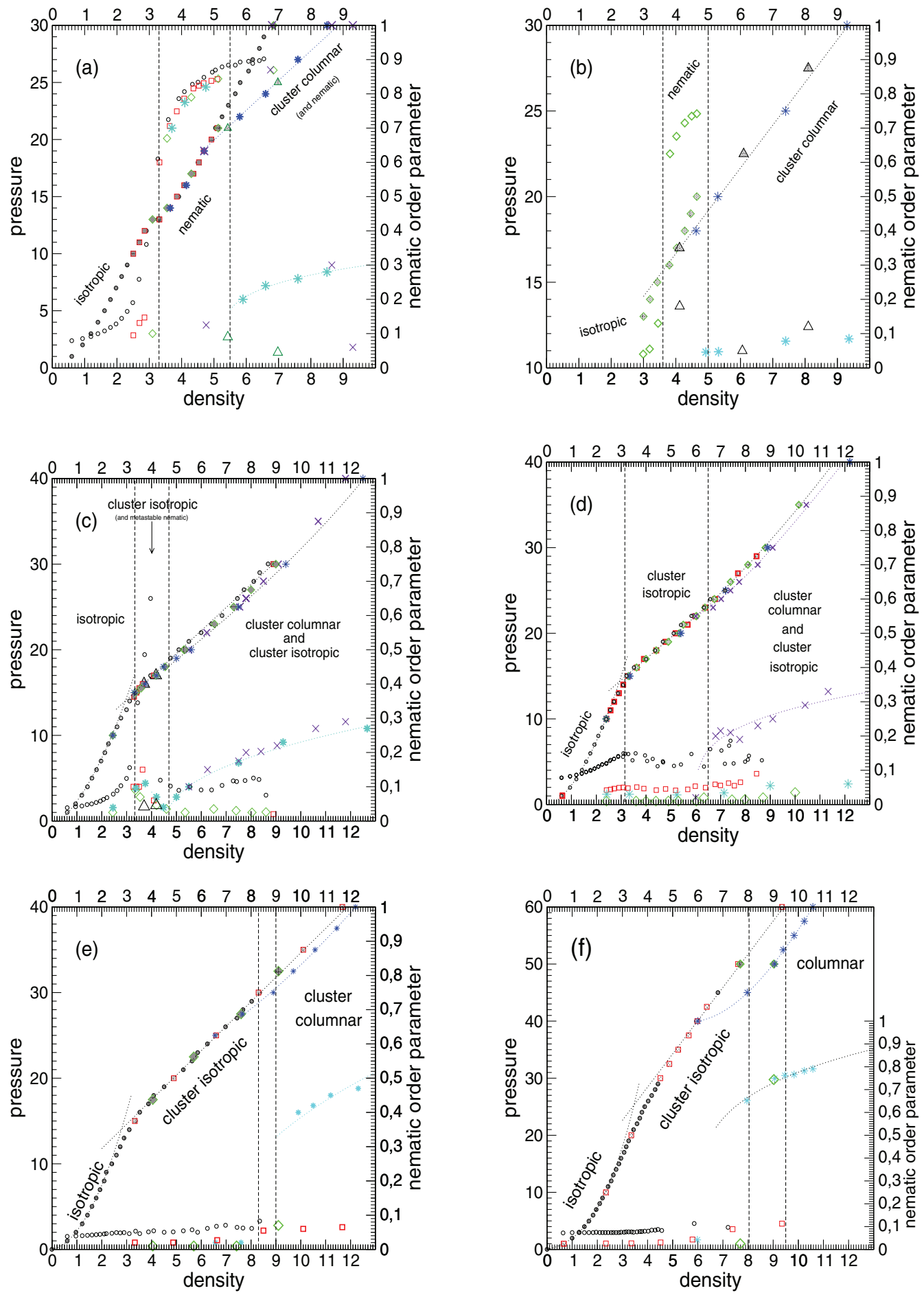

FIG. 3. Equation of state and nematic order parameter. In any panel, the equation of state refers to the left ordinate axis, while the nematic order parameter is on the right ordinate axis. Symbols for the equations of state are full and in darker colors while the corresponding symbols for the nematic order parameter are empty and in lighter colors. Dotted lines crossing equation of state points are polynomial fits as described in the text while dotted lines crossing nematic order parameter points are just a guide to the eye. In general, if not specified, initial configuration belonged to category (i), while it is explicitly written where initial configuration belonged to category (ii). Vertical dashed lines are estimates for the phase transition densities. (a) $R^{*}=2 . \mathrm{N}=125$, black circles; $\mathrm{N}=2000$, red squares; $\mathrm{N}=4096$, green diamonds or triangles; $\mathrm{N}=2000$ and from a $\mathrm{CC}_{\mathrm{h}}$ configuration, blue asterisks; $\mathrm{N}=6000$ or 12000 , indigo crosses. (b) $R^{*}=1.6$. $\mathrm{N}=5000$, green squares or triangles; $\mathrm{N}=4000$ and from a $\mathrm{CC}_{\mathrm{h}}$ configuration, blue asterisks. (c) $R^{*}=1.5 . \mathrm{N}=512$, black circles; $\mathrm{N}=2000$, red squares; $\mathrm{N}=4096$, green diamonds; $\mathrm{N}=12000$ or 16000 , black triangles; $\mathrm{N}=2000$ and from a $\mathrm{CC}_{\mathrm{h}}$ configuration, blue asterisks; $\mathrm{N}=4096$ and from a $C C_{\mathrm{h}}$ configuration,

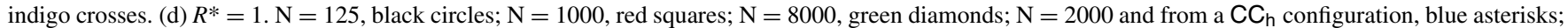
$\mathrm{N}=8000$ and from a CC $\mathrm{h}$ configuration, indigo crosses. (e) $R^{*}=0.7 . \mathrm{N}=512$, black circles; $\mathrm{N}=4096$, red squares; $\mathrm{N}=12000$, green diamonds; $\mathrm{N}=4096$ and from a $\mathrm{CC}_{\mathrm{h}}$ configuration, blue asterisks. (f) $R^{*}=1 / \sqrt{2 \pi} . \mathrm{N}=125$, black circles; $\mathrm{N}=1000$, red squares; $\mathrm{N}=8000$ and from a replica of a $\mathrm{N}=1000$ particle system, green diamonds; $\mathrm{N}=1000$ and from the spontaneously formed $\mathrm{C}_{\mathrm{h}}$ configuration at $P^{*}=60$, blue asterisks. 
(a)

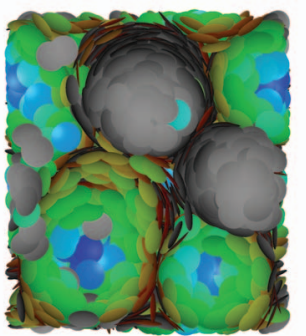

(c)

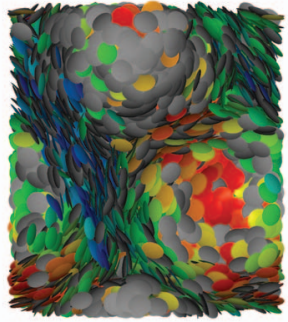

(b)

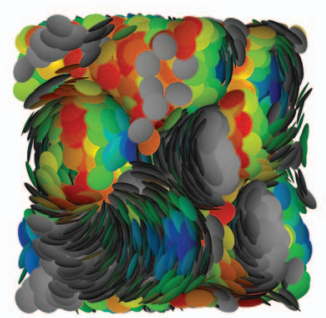

(d)

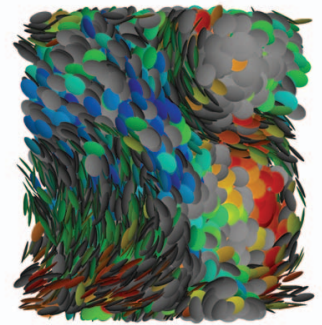

FIG. 4. Images for systems with $R^{*}=2$ at $P^{*}=30[$ (a) and (b)] and $P^{*}=19[(\mathrm{c})$ and $(\mathrm{d})]$.

said at present whether, at a longer range, an overall disordered phase organization such as that in Figs. 4(a) and 4(b) is indeed the one actually favored in place of a more ordered $\mathrm{CC}_{\mathrm{h}}$ structure or instead structures of that type are transient and the system remains locked in to them in its way towards a $\mathrm{CC}_{\mathrm{h}}$ phase organization. Taking a configuration similar to that of Figs. 4(a) and 4(b) and beginning a run with it at $P^{*}=19$ led to the formation of a structure with two relatively rather well defined cluster columns whose interstices are populated by particles that appear as behaving as in a $\mathrm{N}$ phase (Figs. 4(c) and 4(d)). The density of this phase is $\sim 2 \%$ higher than that of a $\mathrm{N}$ phase at the same pressure. The gathering of all these results suggests that, for HSCs, there may exist, at the same value of $P^{*}$, different structures yet sharing very similar values of density. On the basis of these facts, it appears proven that, for $R^{*}=2$, the $\mathrm{N}$ phase becomes unstable at high density owing to the tendency of the particles to aggregate in such a way that they preferentially arrange on the same spherical surface. If one assumes that these aggregates do in turn at last organize to form a $\mathrm{CC}_{\mathrm{h}}$ phase, the transition is a $\mathrm{N}-\mathrm{CC}_{\mathrm{h}}$ phase transition, which may be tentatively located at $P^{*} \sim 21$.

The cases with $R^{*}=1.6$ and 1.5 , were particularly difficult to deal with. For $R^{*}=1.6$ (Fig. 3(b)) and for a system of up to 5000 particles, the $\mathrm{N}$ phase is mechanically stable for $P^{*} \in[16-20]$, but its equilibrium density differs more and more from that of the $\mathrm{CC}_{\mathrm{h}}$ phase as $P^{*}$ increases. By starting a run with a pre-built $\mathrm{N}$ configuration with $\rho^{*}=3.5$ and $\left\langle P_{2}\right\rangle \simeq 0.3$, an I phase was obtained for $P^{*}=13-15$, while for $P^{*}=16,18,19,20$, a $\mathrm{N}$ phase was retained. However, at $P^{*}=17$, the system surprisingly took a completely different pathway and ended up to a complex bunchy structure featuring particles forming round and whirling aggregates, though in the interstices of these particles seem as behaving as in a $\mathrm{N}$ phase. By starting from a configuration of this type, an ordinary $\mathrm{N}$ phase was obtained again at $P^{*}=16$, while starting from a well-equilibrated nematic configuration obtained

(a)

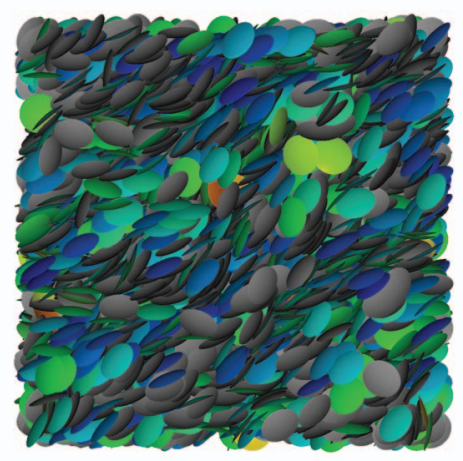

(b)

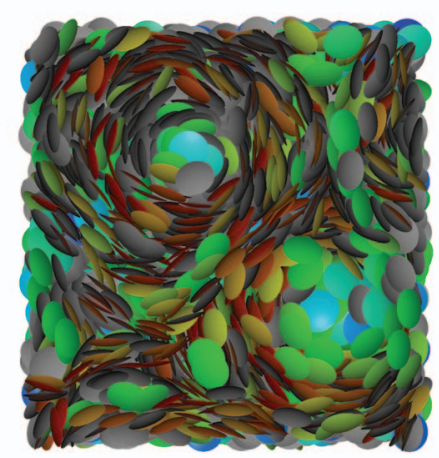

(c)

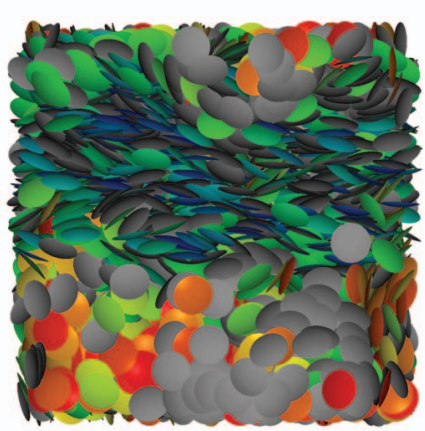

FIG. 5. Images for a system with $R^{*}=1.6$ taken at $P^{*}=17$ : (a) the nematic phase; (b) the phase organisation showing roundish and whirling aggregates, (c) the same configuration as in (b) but from a different perspective, showing a nematic-like stripe in the middle.

at $P^{*}=16$, a $\mathrm{N}$ phase is retained at $P^{*}=17$ (Fig. 5), and for larger values of $P^{*}$ up to 20 . However, by starting with a wellequilibrated nematic configuration obtained at this pressure one run at $P^{*}=22.5$ and another at $P^{*}=27.5$, the systems did not remain nematic. After an initial nematic transient, during which both systems compress and reached a higher orientational order parameter, both then kept compressing but their orientational order parameter begun to decrease. Both systems ended up to a complex bunchy structure of low orientational order parameter, similar to the one obtained for $R^{*}=2$ at $P^{*}=30$ (Figs. 4(a) and 4(b)). All these data suggest that a transition is being approached between the $\mathrm{N}$ phase and a phase showing particle aggregation. This latter should be a $\mathrm{CC}_{\mathrm{h}}$ phase. Starting from a configuration of this type, this phase is preserved for $P^{*} \geq 18$. However, the run at $P^{*}=17$ ended up to a phase exhibiting globular aggregates 


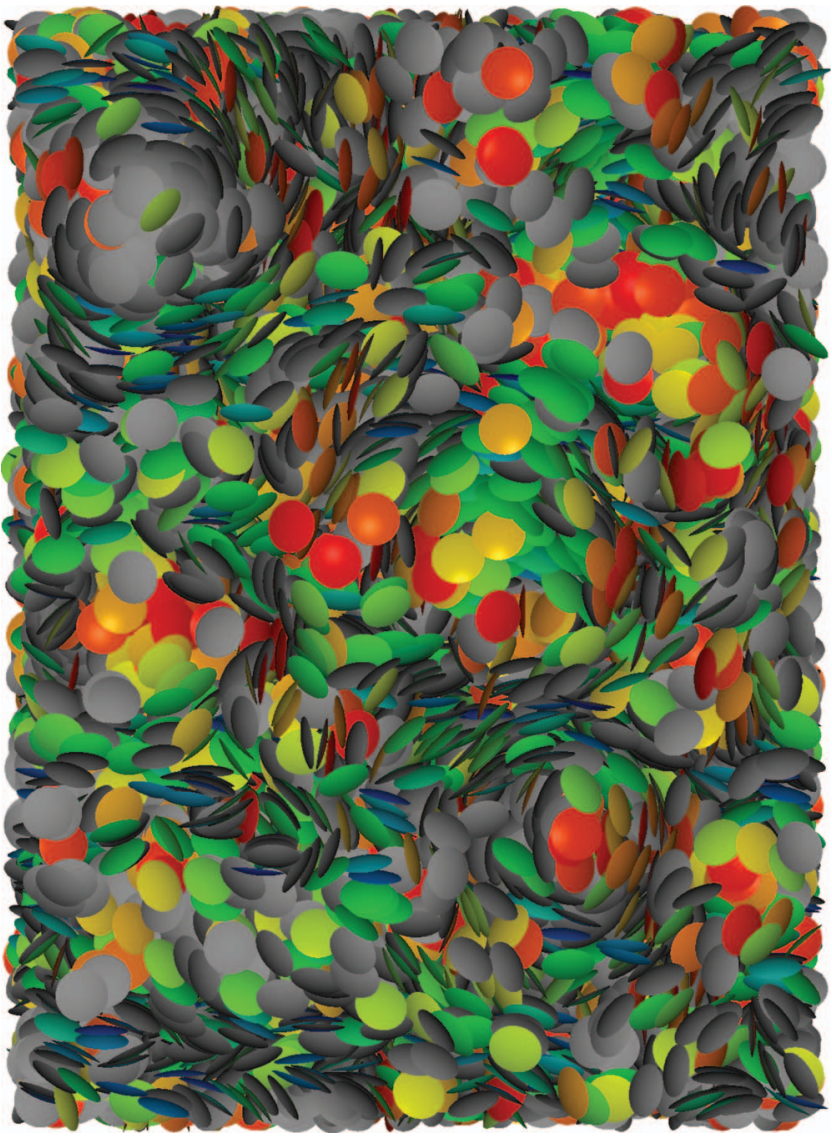

FIG. 6. Typical image of the cluster isotropic phase for $R^{*}=1.5$ at $P^{*}=16$.

with nematic-like interstices, as in the above-mentioned case (Figs. 5(b) and 5(c)). By collecting these results, one can tentatively located the phase transition between the $\mathrm{N}$ and $\mathrm{CC}_{\mathrm{h}}$ phases at $P^{*} \sim 17$.

For $R^{*}=1.5$ (Fig. 3(c)), the $\mathrm{N}$ phase is observed only if small systems, of the order of 500 particles, are handled, while is mechanically unstable for systems of 4000 or more particles. For an intermediate number of particles (2000), there are visible nematic fluctuations close to the density value at which small systems would have an IN phase transition, but a proper $\mathrm{N}$ phase never developed. In the neighborhood of this density value, for larger systems, the EoS shows a kink not accompanied by an upswing in $\left\langle P_{2}\right\rangle$. This is analogous to what observed in Ref. 11 where this feature was ascribed to the development of a cluster isotropic $(\mathrm{Cl})$ phase. In the interval $P^{*} \in[16-18]$ this phase is the only mechanically stable phase. Figure 6 shows a typical image taken at $P^{*}=16$. In runs starting from a $\mathrm{CC}_{\mathrm{h}}$ configuration, this latter type of phase is retained for $P^{*} \geq 19$. One may tentatively locate the $\mathrm{Cl}-\mathrm{CC}_{\mathrm{h}}$ phase transition at this value of $P^{*}$. One can observe though that this phase transition appears characterized by a very small difference in the equilibrium densities of the two branches and distinguishing between them is complicated.

The $\mathrm{Cl}$ phase was first observed in Ref. 11 for a system with $R^{*}=1$. Figure 3(d) confirms those results using a larger number of particles. In addition, a lower bound for the stability of $\mathrm{Cl}$ phase with respect to a $\mathrm{CC}_{\mathrm{h}}$ phase is provided. It (a)

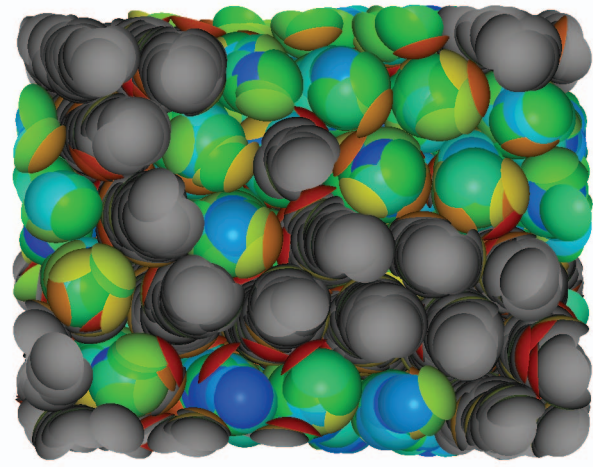

(b)

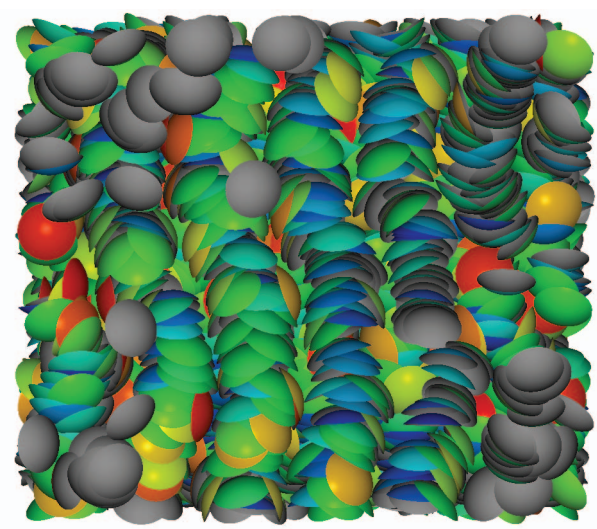

FIG. 7. Top (a) and side (b) views of the hexagonal cluster columnar phase of the system with $R^{*}=0.7$ and at $P^{*}=30$.

was in fact observed that at $P^{*}=22$, a $\mathrm{CC}_{\mathrm{h}}$ phase eventually melted to a $\mathrm{Cl}$ phase after $3 \times 10^{6}$ of $\mathrm{MC}$ cycles in a system of 8000 particles. This is the highest value of $P^{*}$ for which such a melting occurs within a MC run of comparable duration. It may well be possible that the stability of the $\mathrm{Cl}$ phase pushes at larger values of $P^{*}$ : one can observe that also in this case the difference in the equilibrium density between $\mathrm{Cl}$ and $\mathrm{CC}_{\mathrm{h}}$ phases is very small for the range of pressure values examined and this together with the results of Fig. 2 hints that possibility is grounded. None the less, one for now tentatively sets the phase transition pressure at $P^{*} \sim 23$.

The cases with $R^{*}=0.7,0.55,0.45$, and $1 / \sqrt{2 \pi}$ were progressively easier to be handled. There are two intertwined reasons for this. Clusters naturally become of a smaller size and host a smaller number of particles: thus a lesser number of particles is required in the numerical simulations to deal with them. The $\mathrm{Cl}$ EoS branch progressively departs more from the $\mathrm{CC}_{\mathrm{h}}$ EoS branch: this helps distinguish between them. Figures 3(e) and 3(f) show the EoS for $R^{*}=0.7$ and $1 / \sqrt{2 \pi}$, respectively.

In the case of $R^{*}=0.7$, the $\mathrm{CC}_{\mathrm{h}}$ remains at least mechanically stable down to $P^{*}=30$, while at $P^{*}=27.5$ it melted to a $\mathrm{Cl}$ phase after $6 \times 10^{6}$ of $\mathrm{MC}$ cycles. The $\mathrm{Cl}$ phase is rapidly obtained starting from a $\mathrm{CC}_{\mathrm{h}}$ configuration at $P^{*}=$ 25 . This process took $1.2 \times 10^{6}$ of MC cycles. One may tentatively assume that the $\mathrm{Cl}-\mathrm{CC}_{\mathrm{h}}$ phase transition occurs at $P^{*} \sim 30$. Figure 7 provides two views of a $\mathrm{CC}_{\mathrm{h}}$ configuration at this pressure. 

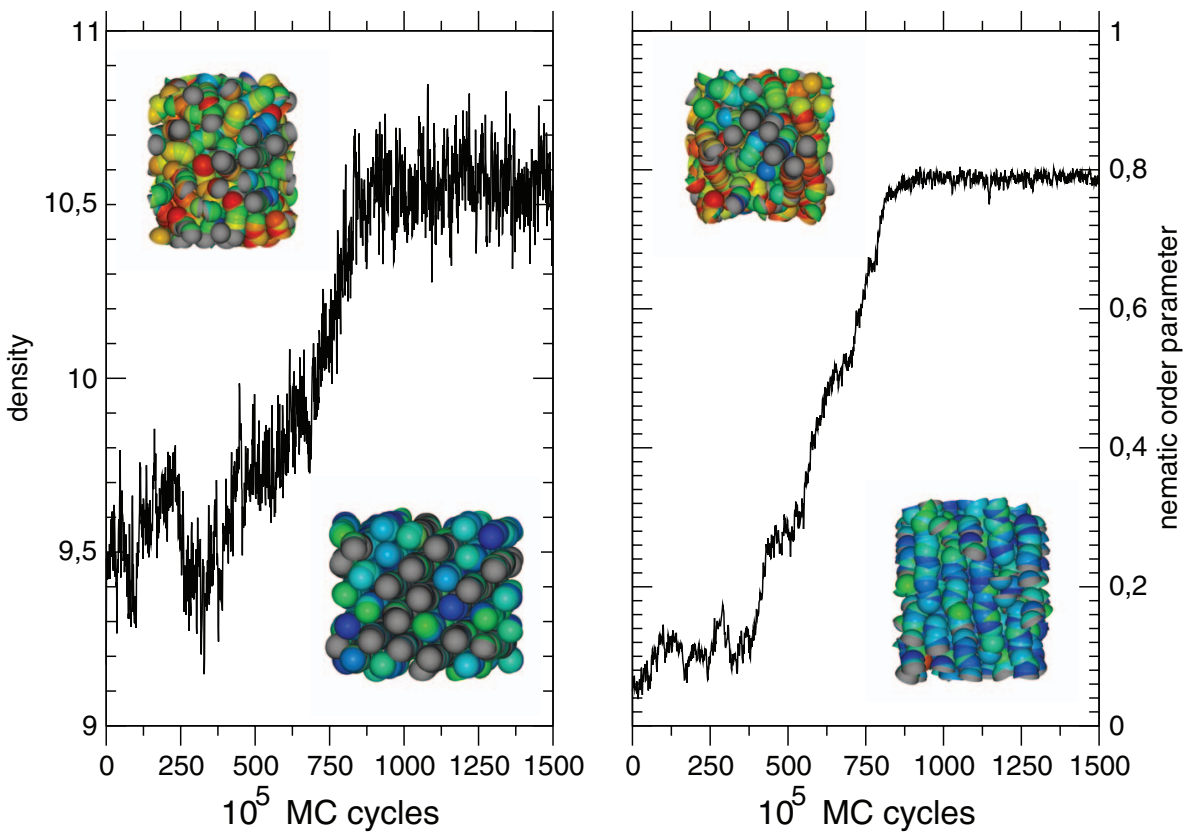

FIG. 8. Spontaneous growth of a columnar order from melt for a system of hard hemispherical cap at $P^{*}=60$. Shown are the density and nematic order parameter along with views of the initial (top) and final (bottom) configurations.

In the case of hard hemispherical caps, the $\mathrm{CC}_{\mathrm{h}}$ reduces to an ordinary $\mathrm{C}_{\mathrm{h}}$ phase. It remains mechanically stable down to $P^{*}=45$ after a run of $50 \times 10^{6}$ of $\mathrm{MC}$ cycles but it melted after $1.5 \times 10^{6}$ of MC cycles at $P^{*}=40$. In Ref. 23(b) the pressure at which a worm-like phase and a columnar phase co-exist was estimated at $P^{*} \simeq 52$ by free-energy calculations. The comparison of this result with the above-mentioned limit of mechanical stability may hint to the latter criterion overestimating the actual $\mathrm{CC}_{\mathrm{h}}$ stability.

The greater simplicity of the hemispherical cap columnar phase made it possible to observe the spontaneous growth of it from an isotropic melt at $P^{*}=60$. This process lasted $100 \times 10^{6}$ of MC cycles for a system of 1000 particles. Figure 8 shows the evolution of both density and nematic order parameter. These numbers indicate how difficult can be the spontaneous growth of columnar order in a spherical cap system. It is plausible that a larger number of MC cycles is needed for systems with a larger value of $R^{*}$, as in these systems the columnar phase is no ordinary, and this, coupled with the larger number of particles required, makes an attempt of spontaneously growing a $\mathrm{CC}_{\mathrm{h}}$ phase from a melt for lens-like particles rather hard at present.

Decreasing particle curvature and compressing the system both contribute to making clusters change from being roundish to filamentous. Figure 9 illustrate this by providing, as an example, two images taken at $P^{*}=17.5$ and 27.5 for the system with $R^{*}=0.7$. This phenomenon may perhaps be more quantitatively appreciated by looking at the behavior of the radial correlation function, $G(X)$, of the parent sphere centers. Figure 10 shows these functions in a fluid system of hemispherical caps. The main peak at small distances indicates the tendency of the centers to clump and its height increases with pressure. The area under this peak is related to the average number of nearest neighbors; this number (plus one) can in turn be interpreted as the average number of particles participating in a cluster $\mathrm{n}_{\mathrm{c}}$. It is interesting to observe that, at low pressure, the height of the second peak at $X=\sqrt{2 / \pi}$ and the shape of the function afterwards match nicely with the curve predicted by Percus-Yevick (PY) integral equation theory for a monodisperse hard sphere system of radius equal to $1 / \sqrt{2 \pi}$ and density $\rho_{\mathrm{c}}=\rho^{*} / \mathrm{n}_{\mathrm{c}}$. This latter value of density may be interpreted as the density of roundish clusters, effectively behaving as hard sphere at lower pressure. The value of $\rho^{*}$ at $P^{*}=20$ is 3.36 and the average number of nearest neighbor, as obtained from the integral of $G(X)$, is 4.47; thus $\mathrm{n}_{\mathrm{c}}=5.47$ and $\frac{\pi}{6} \rho_{\mathrm{c}}\left(\frac{2}{\pi}\right)^{3 / 2} \simeq 0.16$. On increasing $P^{*}$, the correspondence between $G(X)$ and PY result ceases to hold; $G(X)$ is much smoother at $X \sim \sqrt{2 / \pi}$. This is concomitant to the clusters being no longer roundish but filamentous (or worm-like).

Figure 11 shows a sketch of two possible phase diagram topologies that HSCs may exhibit. They have been deduced by gathering together the results obtained so far, with the proviso that the difficulties in drawing conclusions from numerical simulations using a small number of particles are, in the case of the deceptively simple HSC particles, characterized as they are by multiple length scales, particularly exacerbated.

In both parts (a) and (b), it was opted for a representation of the phases in the $1 / R^{*} ; 1 / \rho^{*}$ plane. Data published in Ref. 13 were used for the IN phase transition in hard infinitely thin platelets, while evidence was also acquired on the existence of a first-order transition between a $\mathrm{N}$ and an ordinary $\mathrm{C}_{\mathrm{h}}$ phase in this model system at infinite $P^{*}$ and $\rho^{*} .48$ The data acquired for the fluid-to- $C_{h}$ phase transition for hard hemispherical caps were also used. ${ }^{23(b)}$ For sufficiently large value of $R^{*}$, for which a $\mathrm{N}$ phase is present, the density values of the always weak IN phase transition were estimated from the behavior of $\left\langle P_{2}\right\rangle$. The pressure and consequently the density value(s) of $\mathrm{N}-\mathrm{CC}_{\mathrm{h}}$ or $\mathrm{Cl}-\mathrm{CC}_{\mathrm{h}}$ phase transitions were 
(a)

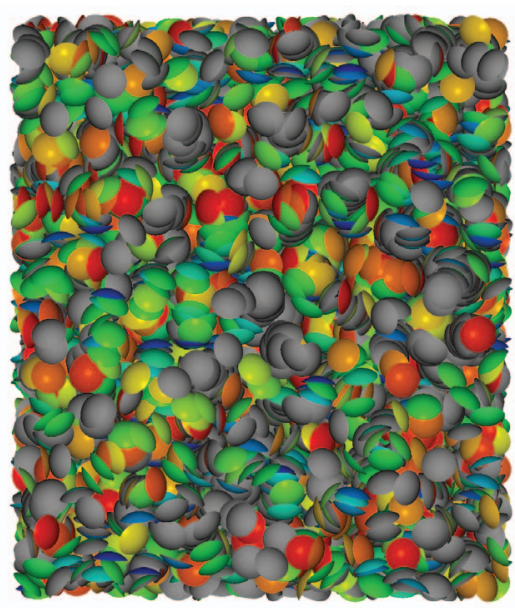

(b)

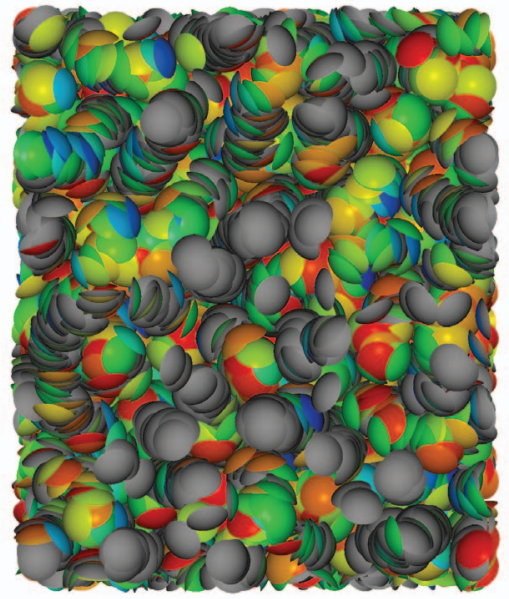

FIG. 9. Images taken for particle systems with $R^{*}=0.7$ at $P^{*}=17.5$ (a) and 27.5 (b).

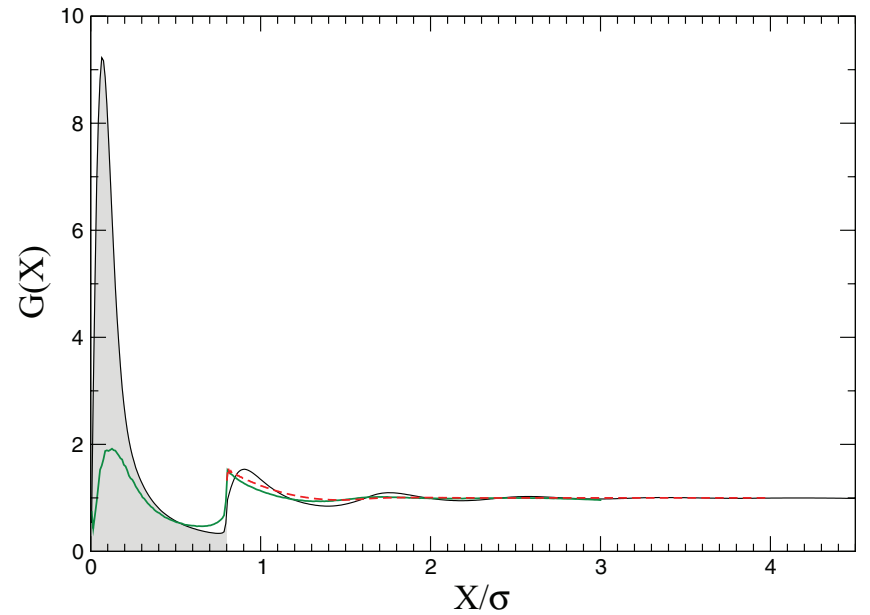

FIG. 10. The function $G(X)$ for a system of hemispherical cap in the fluid phase at $P^{*}=20$ (thick green line) and $P^{*}=50$ (thin black line). For the latter the area under the curve in the interval $[0 ; \sqrt{2 / \pi}]$ is shaded. Shown is also the Percus-Yevick result (dashed red line) for the $g(r)$ of a system of hard spheres at packing fraction 0.16 .
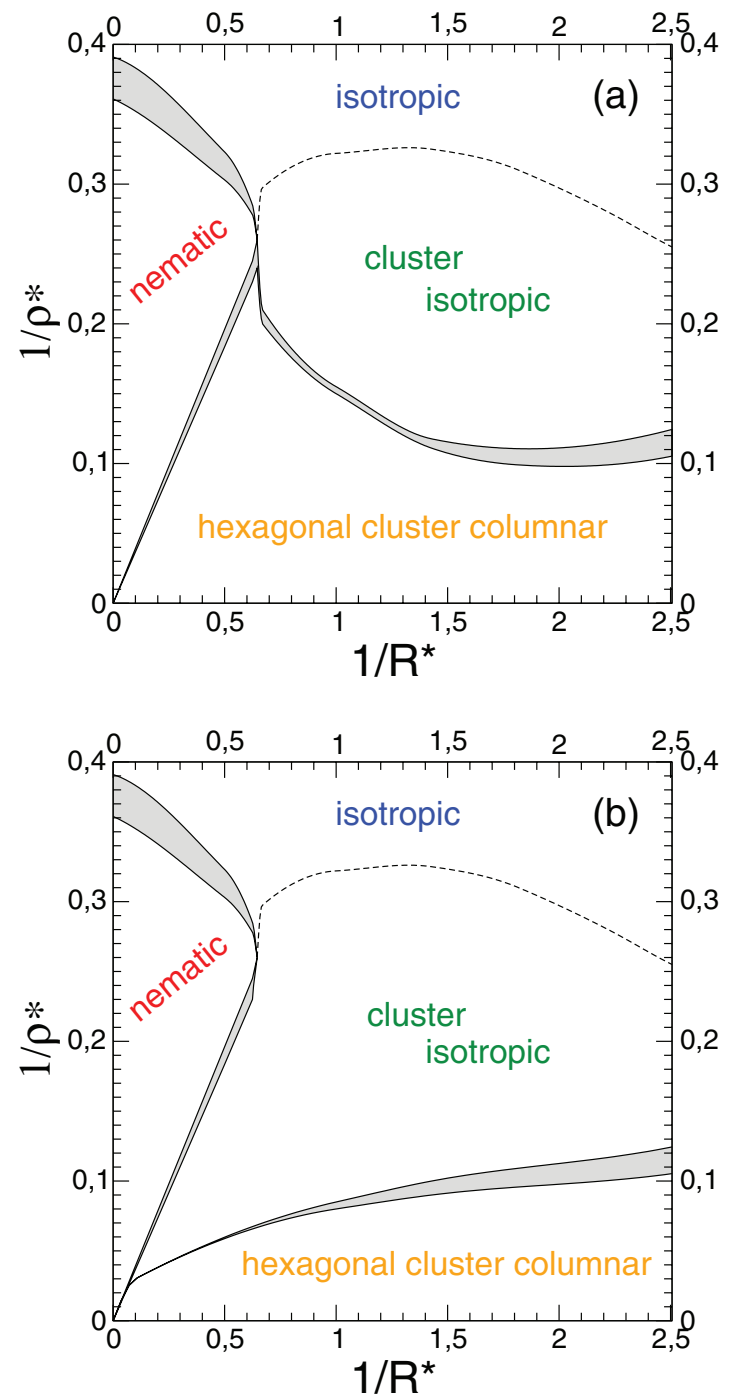

FIG. 11. (a, b) Two tentative phase diagrams of hard spherical caps.

estimated from the mechanical stability of the $\mathrm{CC}_{\mathrm{h}}$ phase. Note that the $\mathrm{N}$ branch of the EoS cannot be linearly fitted for finite values of $R^{*}$, while a linear fit is possible for both $\mathrm{Cl}$ and $\mathrm{CC}_{\mathrm{h}}$ branches within a wide range of pressure values. The density value marking the onset of clustering was arbitrarily set as that density value along the EoS curve having the same pressure value of the intercept of the parabola fitting the low density I phase EoS data with the straight line fitting EoS data corresponding to the $\mathrm{Cl}$ phase, as shown in Fig. 3 .

In both parts (a) and (b), four regions are distinguished, corresponding to the $\mathrm{I}, \mathrm{N}, \mathrm{Cl}$, and $\mathrm{CC}_{\mathrm{h}}$ phases, being the $\mathrm{C}_{\mathrm{h}}$ a particular case of the latter. While the phase diagram topology for small values of $R^{*}$ appears clear, current simulation data are unable to unambiguously disentangle whether, for larger values of $R^{*}$ and on increasing pressure, the $\mathrm{N}$ phase transforms direct to a $C_{h}$ phase, as in Fig. 11(a), or instead it undergoes a transition to a filamentous $\mathrm{Cl}$ phase and then it is the latter that transforms to a $\mathrm{CC}_{\mathrm{h}}$ at higher pressure, as in Fig. 11(b). The ambiguity derives from the inability at present to ascertain whether the complex bunchy and overall disordered structures observed to spontaneously form for $R^{*}=2$ and 1.6 at high pressure are equilibrium or transient 
structures. The phase diagram presented in Fig. 11(a) shares similarities with the generic phase diagram exhibited by flat hard discs as a function of thickness-to-diameter ratio. ${ }^{9}$ In that phase diagram, the $\mathrm{N}$ phase becomes progressively less stable as the particles become thicker and the transition to it from the I phase at a certain point is pre-emptied by a transition direct to a $C_{h}$ phase. The phase diagram presented in Fig. 11(b) corresponds to a more unconventional scenario that present simulation data do not completely rule out.

Whatever is the case, for HSCs the progressive instability of the $\mathrm{N}$ phase is rather caused by their tendency to clump. This is the most basic feature of HSCs together with the fact that this tendency is markedly reflected in the shape of the fluid EoS curve: clusters are equilibrium clusters. This parallels what is occurring in micellizing systems but here is purely entropy driven. This parallelism is further reinforced by the fact that particles having a lens- or bowl-like shape $\left(1 / \sqrt{2 \pi}<R^{*} \lesssim 1.5\right)$ appear to form first roundish aggregates then bunchy filaments and finally hexagonal cluster columnar mesophases.

Further studies are planned with emphasis on the detailed structural characterization of the $\mathrm{Cl}$ phase, as well of interest will be investigating the latter's single-particle and collective dynamics.

The region of the phase diagram corresponding to larger value of $R^{*}$ certainly deserves further dedicated studies. To distinguish between scenario (a) and (b) of Fig. 11 will require systems of very large sizes, much larger than those the author can handle at present (and possibly more advanced MC techniques). In particular, it is the region that would correspond to those two cuspids in the phase diagram of Fig. 11(a) or to the single cuspid in the phase diagram of Fig. 11(b) to be intriguing. Irrespective of which is the actual scenario, it will be of interest to investigate the nature of the IN phase transition in the neighborhood of those values of $R^{*}$ for which the $\mathrm{N}$ is ceasing to be stable and the tendency of particles to clump manifests, as well as the properties of that "fading away" $N$ phase.

In addition, it would be of importance to consider HSClike particles with a finite thickness and check up to what extent cluster phases survive in those cases. One may envisage that, as the particles become thicker, their tendency to clump diminishes at the expense of ordinary phase organizations, whose basic structural unit is a single particle: it should be the (infinitesimal) thinness of HSCs that lets disclose a fluid phase behavior that otherwise would have remained concealed by (partial) crystallization.

One complementary line of research would consist in exploring the phase behavior and properties of those vase-like particles corresponding to values of $R^{*}<1 / \sqrt{2 \pi}$ and focusing on how, structurally and dynamically, they approach the hard sphere $\left(R^{*}=1 / 2 \sqrt{\pi}\right)$ limit.

It is hoped that present results stimulate the synthesis of HSC-like thin colloidal particles and the experimental investigation of their phase behavior, structure, and dynamics. Given today's developments in particle preparation and visualization, this hope appears grounded. The very recent work of Sacanna et al ${ }^{49}$ provides indeed a very important step in this direction.
This research is being supported by the Government of Spain via a Ramón y Cajal research fellowship. The author is grateful to D. Bertolini for his critical reading of the manuscript and to J. S. van Duijneveldt (School of Chemistry, University of Bristol) for his interest in this work and useful discussions. Most of the calculations were run on the computer clusters made available to the author by Professor A. Tani (Dipartimento di Chimica, Università di Pisa); for the purchase of these computer clusters MIUR PRIN 2008 is acknowledged.

${ }^{1}$ G. M. Whitesides and M. Boncheva, Proc. Natl. Acad. Sci. U.S.A. 99, 4769 (2002).

${ }^{2}$ S. C. Glotzer and M. J. Solomon, Nature Mater. 6, 557 (2007).

${ }^{3}$ M. J. Solomon, Curr. Opin. Colloid Interface Sci. 16, 158 (2011).

${ }^{4}$ A. Haji-Akbari, M. Engel, A. S. Keys, X. Zheng, R. G. Petschek, P. PalffyMuhoray, and S. C. Glotzer, Nature (London) 462, 773 (2009); P. Damasceno, M. Engel, and S. C. Glotzer, Science 337, 453 (2012).

${ }^{5}$ U. Agarwal and F. A. Escobedo, Nature Mater. 10, 230 (2011).

${ }^{6}$ J. Henzie, M. Grüwald, A. Widmer-Cooper, P. L. Geissler, and P. Yang, Nature Mater. 11, 131 (2012).

${ }^{7}$ L. Onsager, Ann. N.Y. Acad. Sci. 51, 627 (1949).

${ }^{8}$ M. Hosino, H. Nakano, and H. Kimura, J. Phys. Soc. Jpn. 46, 1709 (1979); ibid. 47, 740 (1979); D. Frenkel, H. N. W. Lekkerkerker, and A. Stroobants, Nature (London) 332, 822 (1988).

${ }^{9}$ J. A. C. Veerman and D. Frenkel, Phys. Rev. A 45, 5632 (1990).

${ }^{10}$ L. J. Ellison, D. J. Michel, F. Barmes, and D. J. Cleaver, Phys. Rev. Lett. 97, 237801 (2006).

${ }^{11}$ G. Cinacchi and J. S. van Duijneveldt, J. Phys. Chem. Lett. 1, 787 (2010).

${ }^{12}$ X. W. Lou, L. A. Archer, and Z. Yang, Adv. Mater. 20, 3987 (2008); K. An and T. Hyeon, Nanotoday 4, 359 (2009).

${ }^{13}$ D. Frenkel and R. Eppenga, Phys. Rev. Lett. 49, 1089 (1982); R. Eppenga and D. Frenkel, Mol. Phys. 52, 1303 (1984).

${ }^{14}$ A. Esztermann, H. Reich, and M. Schmidt, Phys. Rev. E 73, 011409 (2006); D. L. Cheung, L. Anton, M. P. Allen, A. J. Masters, J. Phillips, and M. Schmidt, ibid. 78, 041201 (2008).

${ }^{15}$ L. Harnau and S. Dietrich, in Soft Matter, edited by G. Gompper and M. Schick (Wiley, Berlin, 2007), Vol. 3, pp. 159-311; L. Harnau, Mol. Phys. 106, 1975 (2008).

${ }^{16}$ M. P. Allen, G. T. Evans, D. Frenkel, and B. M. Mulder, Adv. Chem. Phys. 86, 1 (1993).

${ }^{17}$ C. Zannoni, J. Mater. Chem. 11, 2637 (2001).

${ }^{18}$ C. Avendaño, C. M. Liddell Watson, and F. A. Escobedo, Soft Matter 9, 9153 (2013).

${ }^{19}$ T. Niori, T. Sekine, J. Watanabe, T. Furukawa, and H. Takezoe, J. Mater. Chem. 6, 1231 (1996).

${ }^{20}$ B. R. Acharya, A. Primak, T. J. Dingemans, E. T. Samulski, and S. Kumar, Pramana 61, 231 (2003); L. A. Madsen, T. J. Dingemans, M. Nakata, and E. T. Samulski, Phys. Rev. Lett. 92, 145505 (2004); B. R. Acharya, A. Primak, and S. Kumar, ibid. 92, 145506 (2004).

${ }^{21}$ O. Francescangeli and E. T. Samulski, Soft Matter 6, 2413 (2010).

${ }^{22}$ M. Ricci, R. Berardi, and C. Zannoni, Soft Matter 4, 2030 (2008).

${ }^{23}$ (a) M. Marechal, R. J. Kortschot, A. F. Demirörs, A. Imhof, and M. Dijkstra, Nano Lett. 10, 1907 (2010); (b) M. Marechal and M. Dijkstra, Phys. Rev. E 82, 031405 (2010)

${ }^{24}$ J. Malthête and A. Collet, J. Am. Chem. Soc. 109, 7544 (1987); G. Cometti, E. Dalcanale, A. Du Vosel, and A. M. Levelut, J. Chem. Soc., Chem. Commun. 1990, 163; G. Cometti, E. Dalcanale, A. Du Vosel, and A. M. Levelut, Liq. Cryst. 11, 93 (1992); B. Xu and T. M. Swanger, J. Am. Chem. Soc. 115, 1159 (1993).

${ }^{25}$ M. Sawamura, K. Kasai, Y. Matsuo, K. Kanie, T. Kato, and E. Nakamura, Nature (London) 419, 702 (2002).

${ }^{26}$ C. I. Zoldesi, C. A. van Walree, and A. Imhof, Langmuir 22, 4343 (2006).

${ }^{27}$ J. C. Love, B. D. Gates, D. B. Wolfe, K. E. Paul, and G. M. Whitesides, Nano Lett. 2, 891 (2002).

${ }^{28}$ Y. T. Wu and J. S. Siegel, Chem. Rev. 106, 4843 (2006); V. M. Tsefrikas and L. T. Scott, ibid. 106, 4868 (2006); T. Kawase and H. Kurata, ibid. 106, 5250 (2006); A. Sygula, Eur. J. Org. Chem. 2011, 1611.

${ }^{29}$ H. Tanaka, Phys. Rev. E 62, 6968 (2000).

${ }^{30}$ J. Groenewold and W. K. Kegel, J. Phys. Chem. B 105, 11702 (2001). 
${ }^{31}$ P. N. Segrè, V. Prasad, A. B. Schofield, and D. A. Weitz, Phys. Rev. Lett. 86, 6042 (2001).

${ }^{32}$ F. Sciortino, S. Mossa, E. Zaccarelli, and P. Tartaglia, Phys. Rev. Lett. 93, 055701 (2004).

${ }^{33}$ A. I. Campbell, V. J. Anderson, J. S. van Duijneveldt, and P. Bartlett, Phys. Rev. Lett. 94, 208301 (2005).

${ }^{34}$ F. Sciortino, A. Giacometti, and G. Pastore, Phys. Rev. Lett. 103, 237801 (2009).

${ }^{35}$ W. Klein, H. Gould, R. A. Ramos, I. Clejan, and A. I. Melcuk, Physica A 205, 738 (1994); B. M. Mladek, D. Gottwald, G. Kahl, M. Neumann, and C. N. Likos, Phys. Rev. Lett. 96, 045701 (2006); C. N. Likos, B. M. Mladek, D. Gottwald, and G. Kahl, J. Chem. Phys. 126, 224502 (2007).

${ }^{36}$ M. Kléman and J. F. Sadoc, J. Phys. Lett. 40, 569 (1979); J. F. Sadoc and R. Mosseri, Geometrical Frustration (Cambridge University Press, 1999).

${ }^{37}$ W. M. Gelbart and A. Ben Shaul, J. Phys. Chem. 100, 13169 (1996).

${ }^{38}$ J. N. Israelachvili, Intermolecular and Surface Forces (Academic Press, San Diego, 2011).

${ }^{39}$ W. W. Wood, J. Chem. Phys. 48, 415 (1968); H. F. King, ibid. 57, 1837 (1972)
${ }^{40}$ M. P. Allen and D. J. Tildesley, Computer Simulation of Liquids (Oxford University Press, 1987).

${ }^{41}$ M. He and P. Siders, J. Phys. Chem. 94, 7280 (1990).

${ }^{42}$ R. D. Mountain and D. Thirumalai, Physica A 210, 453 (1994).

${ }^{43}$ W. H. Press, S. A. Teukolsky, W. T. Vetterling, and B. P. Flannery, Numerical Recipes: The Art of Scientific Computing (Cambridge University Press, Cambridge, 1996).

${ }^{44}$ M. Matsumoto and T. Nishimura, ACM Trans. Model. Comput. Simul. 8, 3 (1998).

${ }^{45}$ See http://qmga.sourceforge.net/ for QMGA program; A. T. Gabriel, T. Meyer, and G. Germano, J. Chem. Theory Comput. 4, 468 (2008).

${ }^{46}$ J. Veilliard-Baron, Mol. Phys. 28, 809 (1974).

${ }^{47}$ In all snapshots, top parts of particles were color coded according to the orientation they have in the laboratory frame of reference having the nematic director pointing along the $\mathrm{Z}$ axis, while their bottom parts were left in gray.

${ }^{48}$ M. Bates and D. Frenkel, Phys. Rev. E 57, 4824 (1998).

${ }^{49}$ S. Sacanna, M. Korpics, K. Rodriguez, L. Colón-Meléndez, S. H. Kim, D. J. Pine, and G. R. Yi, Nature Commun. 4, 1688 (2013). 